\title{
A Teleophthalmological intervention to integrate diabetic and eye clinics to deliver care for diabetic retinopathy
}

\author{
M. De Silva ${ }^{1}$, V. Gurusamy ${ }^{2}$, R.B. Marasinghe ${ }^{3}$ \\ 1 Post Graduate institute of Medicine, University of Colombo, Sri Lanka \\ 2 Colombo South Teaching Hospital, Kalubowila, Sri Lanka \\ 3 Faculty of Medical Sciences, University of Sri Jayewardenepura, Sri Lanka \\ *malid8g@gmail.com \\ https://orcid.org/0000-0001-8331-8257
}

\begin{abstract}
Introduction: Diabetes and its complications add extra burden to the global disease burden. Diabetic retinopathy (DR) already accounts for $4 \%$ of blindness worldwide. Though screening of all diabetics for DR is advocated, only $60 \%$ global coverage is met. High patient volume, patient factors and system factors contribute to this limitation. Teleophthalmology is a recognized method to overcome some of these limiting factors. This study aimed to design \& develop a teleophthalmology platform suited to a local setting and to evaluate the post implementation outcomes.
\end{abstract}

Methods: The study adopted action-based research approach. A tertiary care hospital was selected with an established integrated care process for DR screening. Global teleophthalmology systems and their implementation strategies were reviewed. The requirements were gathered through key informant interviews i.e. clinicians. A teleophthalmology platform was developed and deployed to connect the diabetic clinic and the eye clinic for the purpose of evaluating the fundal images as a part of DR screening. The intervention was evaluated based on patient factors, comparing using the independent samples $t$ test between the two samples screened for DR, with and without the teleophthalmology intervention.

Results: 40 patients each were screened for the two samples with and without the aid of the teleophthalmology intervention. The average age of the participants were 62.6 years \& 53.6 years while $76 \%$ were females and $42 \%$ males, respectively in the two samples. Patients screened for DR with the aid of the teleophthalmology platform showed reduced number of visits $(p=0.001)$, reduced waiting time $(p<0.017)$ and higher satisfaction $(p<0.002)$. The overall cost incurred by patients per visit $(p=0.36)$ and for completion of DR screening ( $p=0.91$ ) was not significantly different.

Conclusions: Adoption of teleophthalmology improves the patient waiting time, number of visits and satisfaction. This could be considered as an effective and feasible intervention to integrate into an established clinical setup for managing diabetic retinopathy.

Key words: Telemedicine, Teleophthalmology, Diabetic retinopathy, Fundal imaging, Patient convenience 